\title{
Associations of six adiposity-related markers with incidence and mortality from 24 cancers-findings from the UK Biobank prospective cohort study
}

Solange Parra-Soto ${ }^{1,2}$, Emma S. Cowley², Leandro F. M. Rezende ${ }^{3}$, Catterina Ferreccio ${ }^{4,5}$, John C. Mathers ${ }^{6 \dagger}$, Jill P. Pell ${ }^{1+}$, Frederick K. Ho ${ }^{1+}$ and Carlos Celis-Morales ${ }^{1,2,7,8^{*+}}$

\begin{abstract}
Background: Adiposity is a strong risk factor for cancer incidence and mortality. However, most of the evidence available has focused on body mass index (BMI) as a marker of adiposity. There is limited evidence on relationships of cancer with other adiposity markers, and if these associations are linear or not. The aim of this study was to investigate the associations of six adiposity markers with incidence and mortality from 24 cancers by accounting for potential non-linear associations.

Methods: A total of 437,393 participants (53.8\% women; mean age 56.3 years) from the UK Biobank prospective cohort study were included in this study. The median follow-up was 8.8 years (interquartile range 7.9 to 9.6) for mortality and 9.3 years (IQR 8.6 to 9.9) for cancer incidence. Adiposity-related exposures were BMI, body fat percentage, waist-hip ratio, waist-height ratio, and waist and hip circumference. Incidence and mortality of 24 cancers sites were the outcomes. Cox proportional hazard models were used with each of the exposure variables fitted separately on penalised cubic splines.

Results: During follow-up, 47,882 individuals developed cancer and 11,265 died due to cancer during the follow-up period. All adiposity markers had similar associations with overall cancer incidence. BMI was associated with a higher incidence of 10 cancers (stomach cardia (hazard ratio per 1 SD increment 1.35, (95\% Cl 1.23; 1.47)), gallbladder $(1.33(1.12 ; 1.58)$ ), liver (1.27 (1.19; 1.36)), kidney (1.26 (1.20; 1.33)), pancreas $(1.12(1.06 ; 1.19))$, bladder $(1.09(1.04 ; 1.14))$, colorectal $(1.10(1.06 ; 1.13))$, endometrial $(1.73(1.65 ; 1.82))$, uterine $(1.68(1.60 ; 1.75))$, and breast cancer $(1.08(1.05 ; 1.11)))$ and overall cancer $(1.03(1.02$; 1.04)). All these associations were linear except for breast cancer in postmenopausal women. Similar results were observed when other markers of central and overall adiposity were used. For mortality, nine cancer sites were linearly associated with $\mathrm{BMl}$ and eight with waist circumference and body fat percentage.
\end{abstract}

Conclusion: Adiposity, regardless of the marker used, was associated with an increased risk in 10 cancer sites.

Keywords: Obesity, Body mass index, Waist circumference, Body fat, Cancer, UK Biobank

\footnotetext{
* Correspondence: Carlos.Celis@glasgow.ac.uk

${ }^{\dagger}$ John C. Mathers, Jill P. Pell, Frederick K. Ho and Carlos Celis-Morales contributed equally to this study and are joint-senior authors.

'Institute of Health and Wellbeing, University of Glasgow, Glasgow G12 8RZ,

UK

${ }^{2}$ Institute of Cardiovascular and Medical Sciences, University of Glasgow,

Glasgow G12 8TA, UK

Full list of author information is available at the end of the article
}

C C The Author(s). 2021 Open Access This article is licensed under a Creative Commons Attribution 4.0 International License, which permits use, sharing, adaptation, distribution and reproduction in any medium or format, as long as you give appropriate credit to the original author(s) and the source, provide a link to the Creative Commons licence, and indicate if changes were made. The images or other third party material in this article are included in the article's Creative Commons licence, unless indicated otherwise in a credit line to the material. If material is not included in the article's Creative Commons licence and your intended use is not permitted by statutory regulation or exceeds the permitted use, you will need to obtain permission directly from the copyright holder. To view a copy of this licence, visit http://creativecommons.org/licenses/by/4.0/. The Creative Commons Public Domain Dedication waiver (http://creativecommons.org/publicdomain/zero/1.0/) applies to the data made available in this article, unless otherwise stated in a credit line to the data. 


\section{Background}

Currently, $67 \%$ of men and $62 \%$ of women are overweight or obese in the UK. Obesity has strong association with increased incidence of, and premature mortality from, some types of cancer $[1,2]$. A recent report by the World Cancer Research Fund (WCRF) summarises the evidence showing that high BMI is associated with higher risk of 12 cancers, including colorectal, breast in postmenopausal women, oesophageal, pancreatic, liver, kidney, oral, pharynx and larynx, stomach cardia, gallbladder, ovarian, (advanced) prostate, and womb cancers [3]. However, the WCRF report also highlighted the lack of evidence regarding the association of cancer with other markers of adiposity (i.e. central adiposity and body fat).

Although previous studies have reported the association of several cancer sites with different markers of adiposity [2, 4, 5], most of these studies have been conducted in Asian populations [6, 7], Lee et al. reported the associations of 18 cancers with waist circumference (WC) in 22.9 million Korean adults [7]. Similarly, Wang et al. reported the associations of four markers of adiposity including BMI, WC, waist-to-hip ratio (WHR), and body fat percentage (BF\%) with 15 cancers in the China Kadoorie Biobank [6]. Evidence derived from white or British populations has focused mainly on a small number of cancer sites (i.e. breast, colon, endometrium, and prostate) [8-11], or has been restricted to BMI as a marker of adiposity [2, 4, 5]. In 2014, Bhaskaran et al. [2] reported that BMI was associated with 17 cancers in 5.2 million British adults. This study also highlighted the need for further evidence for other adiposity markers since measures of body fat distribution, such as central obesity and body fat might be stronger determinants of specific cancer sites than BMI [12], as observed for other health outcomes such as cardiovascular diseases [13]. Moreover, most of the evidence available to date have assumed a linear association between markers of adiposity and cancer risk from most common sites (colorectal, breast cancer, liver, kidney, and gallbladder) $[4,12]$, with a limited number of studies investigating non-linear association $[2,14,15]$. To address these limitations, we used data from the UK Biobank cohort, a large prospective cohort study, to investigate the associations of six adiposity markers with incidence and mortality from 24 cancers by accounting for potential non-linear associations.

\section{Methods}

\section{Study design}

UK Biobank recruited more than 500,000 participants (aged 37-73 years, 56.3\% were women) between 2006 and 2010 [16]. Participants attended one of 22 assessment centres across England, Scotland, and Wales, where they completed a self-administered, touchscreen questionnaire and face-to-face interviews [17, 18]. After excluding participants with a prevalent cancer diagnosis at baseline $(n=41,460)$, those with missing data for exposures and covariates $(n=21,064)$, and participants who were classified as underweight ( $n=2629), 437,393$ participants were finally included in the study. The outcomes defined for this study were incidence and mortality of overall cancer and 24 specific cancers. Of the 24 cancers, 17 were relevant to both men and women, two were specific to men (testicular and prostate cancer), and five were specific to women (breast, endometrium, uterine, cervix and ovary). The exposures were six adiposity-related markers, including BMI, WC, WHR, waist-to-height ratio (WHtR), hip circumference $(\mathrm{HC})$, and $\mathrm{BF} \%$. The covariates were sociodemographic factors (age, ethnicity, education, and Townsend deprivation), smoking status, dietary intake (red meat, processed meat, fruit and vegetables, oily fish, and alcohol), physical activity, and sedentary behaviour. Additional cancerspecific covariates were added for women-related cancer (hormonal replacement, ages at first live birth, last live birth, and at menarche). Additionally, sun exposition was added as a covariate for melanoma cancer, and for lung, oesophageal, and oral cancer, we restricted the analysis to never smoker only. Association between adiposity markers and cancer mortality is likely the combined effect of adiposity's association with incident cancer, and adiposity's association with cancer fatality among cancer patients.

\section{Procedures}

Date of death was obtained from death certificates held within the National Health Service Information Centre (England and Wales) and the National Health Service Central Register Scotland (Scotland). Date and cause of hospital admissions were obtained through record linkage to Health Episode Statistics (England and Wales) and Scottish Morbidity Records (Scotland). Detailed information about the linkage procedures can be found at http://content.digital.nhs. $\mathrm{uk} /$ services. At the time of analysis, mortality data were available up to 01 June 2020. Mortality analysis was therefore censored at this date or date of death, whichever occurred earlier. Hospital admission data were available until 31 March 2017 for Scotland and Wales and until 01 June 2020 for England, resulting in analyses of incident outcomes being censored at this date or the date of relevant hospitalisation or death, whichever occurred earlier. We defined incident cancer as fatal or nonfatal events. The International Classification of Diseases, 10th revision (ICD-10), was used to define the following 27 cancers: overall cancer (C00-C97, 
D37, D48), oral (lip, pharynx and larynx) (C00-C14), oesophagus (C15) upper oesophagus (C15.0, 15.1, 15.3, and 15.4), stomach (C16) stomach cardia (C16.0), stomach non cardia (C16.1-16.6), colorectal (C18, C19, and C20), colon proximal (C 18.0-18.5), colon distal (C18.6, C18.7), colon (C18.0-C18.9), rectum (C19-C20), liver (C22), gallbladder (C23), pancreas (C25), lung (C34), malignant melanoma (C43), breast (C50), uterine (C54-C55), cervix (C53), endometrium (C54), ovary (C56), prostate (C61), testis (C62), kidney (C64-C65), bladder (C67), brain (C71), thyroid (C73), lymphatic and haematopoietic tissue (C81C96), non-Hodgkin lymphoma (C82-C85), multiple myeloma (C90), and leukaemia (C91-C95).

The exposures were six adiposity-related markers (BMI, WC, WHR, WHtR, HC, and BF\%) measured by trained staff using standardised protocols across the assessment centres at baseline. Height was measured to the nearest centimetre, using a Seca 202 stadiometer, and body weight to the nearest $0.1 \mathrm{~kg}$, using a Tanita BC-418 body composition analyser. BMI was calculated as weight $(\mathrm{kg})$ divided by height $(\mathrm{m})$ squared and classified into the following categories: underweight $\left(<18.5 \mathrm{~kg} / \mathrm{m}^{2}\right)$, normal weight (18.5 to $\left.<25 \mathrm{~kg} / \mathrm{m}^{2}\right)$, overweight $\left(25\right.$ to $\left.<30 \mathrm{~kg} / \mathrm{m}^{2}\right)$, and obese $\left(>30 \mathrm{~kg} / \mathrm{m}^{2}\right)$ [19].

BF\% was measured using the Tanita BC-418 MA body composition analyser (fat mass divided by the total body mass).

The natural indent was used to measure WC (the umbilicus was used if the natural indent could not be observed) and used to determine central obesity (WC $\geq 88$ $\mathrm{cm}$ for women and WC $\geq 102 \mathrm{~cm}$ for men). HC was recorded at the widest part of the hips. WHR and WHtR are the ratios of the waist-to-hip circumference and waist circumference to height, respectively.

Age, sex, ethnicity, smoking status, diet (portions of fruits and vegetables, red and processed meat, and oily fish) and alcohol intake (daily, 2-4 times a week, once or twice a week, 1-3 time a month, special occasions and never), sun exposition (do not go out in the sunshine, rarely, sometimes, most of the time, always), and female-specific factors were self-reported at the baseline assessment by touch-screen questionnaire. Townsend area deprivation index was derived from the postcode of residence using aggregated data on unemployment, car and homeownership, and household overcrowding [20]. Educational qualification was self-reported. Physical activity level over a typical week was self-reported using the International Physical Activity Questionnaire and reported as metabolic equivalent of task (MET) per week [21]. Time spent on discretionary sedentary behaviours was derived from the questionnaire and included time spent in front of a TV or computer or driving during leisure time. Further details of these measurements can be found in the UK Biobank online protocol (http:// www.ukbiobank.ac.uk).

\section{Statistical analyses}

Cox proportional hazard models were used to estimate hazard ratios (HR) and 95\% confidence intervals for each adiposity marker (BMI, WC, BF\%, WHR, WHtR, and $\mathrm{HC}$ ) separately with incidence and mortality for 24 cancers and all-cause cancer. Duration of follow-up was used as the timeline variable. The exposure variables were fitted separately on penalised cubic splines to investigate non-linear associations between each adiposity exposure and the outcomes. Penalised spline is a variation of basis spline [22]. Non-linearity was tested by likelihood ratio tests. To compare the associations between cancer across different adiposity markers, all adiposity exposures were standardised by sex and HR were expressed per 1-standard deviation increment (1-SD was equivalent to BMI units of 4.2 and $5.1 \mathrm{~kg} / \mathrm{m}^{2}$, WC 11.3 and $12.5 \mathrm{~cm}$, WHR 0.07 and 0.07 , WHtR 6.5 and 7.9, HC 7.6 and $10.4 \mathrm{~cm}, \mathrm{BF} \% 5.8$ and $6.9 \%$, and BFI 2.6 and $3.8 \mathrm{~kg} / \mathrm{m}^{2}$ for men and women, respectively). Participants with prevalent cancer at the baseline assessment were excluded from the study $(n=41,406)$. Underweight participants were also excluded from the study $(n=2629)$. In addition, a landmark analysis was performed to reduce the potential for reverse causality, with follow-up commencing 2 years after recruitment. The association between adiposity and oesophageal, oral, and lung cancer was restricted to participants who reported being never smokers, to avoid reverse causation bias. For breast cancer, all analyses were stratified by menopausal status. Additional sensitivity analyses were performed including underweight people and adding height as a covariate.

Population attributable fractions (PAFs), assuming causality, were calculated based on the BMI distribution of Health Surveys of England, Scotland, and Wales in 2018 [23-25] and the HRs derived from this study using the standard formula with $95 \%$ confidence interval (CI) and $P$ values estimated using bootstrapping (formula shown in Additional file 1: Figure S1) [26].

To compare cancer risk discrimination between BMI and the remaining five adiposity markers, we calculated Harrell's C-index (the probability of concordance between observed and predicted responses) for a model that included the adiposity marker and covariates (age, ethnicity, deprivation, education, smoking, alcohol consumption, intakes of fruit and vegetables, red and processed meat, oily fish, physical activity, and sedentary behaviours). The model with BMI was defined as baseline model. The $\mathrm{C}$-indices of the baseline model and the $\mathrm{C}$-index difference between other adiposity model and the baseline model were reported. The variance of the $\mathrm{C}$-indices was calculated using the formula as described 
Table 1 Cohort baseline characteristics

\begin{tabular}{|c|c|c|c|c|}
\hline & Normal weight & Overweight & Obese & Overall \\
\hline$n$ & $143,460(32.8 \%)$ & $187,563(42.9 \%)$ & $106,370(24.3 \%)$ & 437,393 \\
\hline Age, mean (SD) & $55.4(8.22)$ & $56.7(8.07)$ & $56.6(7.90)$ & $56.3(8.10)$ \\
\hline \multicolumn{5}{|l|}{ Sex } \\
\hline Females & $92,922(64.8 \%)$ & 87,097 (46.4\%) & $55,246(51.9 \%)$ & $235,265(53.8 \%)$ \\
\hline Males & $50,538(35.2 \%)$ & $100,466(53.6 \%)$ & $51,124(48.1 \%)$ & $202,128(46.2 \%)$ \\
\hline \multicolumn{5}{|l|}{ Townsend deprivation index } \\
\hline Lower deprivation & $51,511(35.9 \%)$ & $65,530(34.9 \%)$ & $30,740(28.9 \%)$ & $147,781(33.8 \%)$ \\
\hline Middle deprivation & $48,183(33.6 \%)$ & $63,918(34.1 \%)$ & $34,366(32.3 \%)$ & $146,467(33.5 \%)$ \\
\hline Higher deprivation & $43,766(30.5 \%)$ & 58,115 (31.0\%) & $41,264(38.8 \%)$ & $143,145(32.7 \%)$ \\
\hline \multicolumn{5}{|l|}{ Education } \\
\hline College or University degree & $64,263(44.8 \%)$ & $69,351(37.0 \%)$ & $32,442(30.5 \%)$ & $166,056(38.0 \%)$ \\
\hline A levels/AS levels or equivalent & $17,455(12.2 \%)$ & 20,738 (11.1\%) & $11,116(10.5 \%)$ & $49,309(11.3 \%)$ \\
\hline O levels/GCSEs or equivalent & $29,336(20.4 \%)$ & $40,223(21.4 \%)$ & $23,510(22.1 \%)$ & $93,069(21.3 \%)$ \\
\hline SEs or equivalent/NVQ or HND or HNC or equivalent & $13,885(9.7 \%)$ & $23,548(12.6 \%)$ & $15,352(14.4 \%)$ & $52,785(12.1 \%)$ \\
\hline Missing & $18,521(12.9 \%)$ & $33,703(18.0 \%)$ & $23,950(22.5 \%)$ & $76,174(17.4 \%)$ \\
\hline \multicolumn{5}{|l|}{ Ethnicity } \\
\hline White & $136,331(95.0 \%)$ & $177,574(94.7 \%)$ & $99,866(93.9 \%)$ & $413,771(94.6 \%)$ \\
\hline Mixed & $2101(1.5 \%)$ & $2703(1.4 \%)$ & $1741(1.6 \%)$ & $6545(1.5 \%)$ \\
\hline South Asian & $2830(2.0 \%)$ & $3965(2.1 \%)$ & $1869(1.8 \%)$ & $8664(2.0 \%)$ \\
\hline Black & $1327(0.9 \%)$ & $2905(1.5 \%)$ & $2813(2.6 \%)$ & $7045(1.6 \%)$ \\
\hline Chinese & $871(0.6 \%)$ & $416(0.2 \%)$ & $81(0.1 \%)$ & $1368(0.3 \%)$ \\
\hline Height $(m)$, mean (SD) & $1.68(0.08)$ & $1.69(0.09)$ & $1.68(0.09)$ & $1.69(0.09)$ \\
\hline Weight (kg), mean (SD) & $64.7(8.47)$ & $78.6(9.63)$ & $95.9(14.3)$ & $78.2(15.8)$ \\
\hline Waist circumference $(\mathrm{cm})$, mean (SD) & $78.6(8.10)$ & $91.0(8.36)$ & $105(11.0)$ & $90.3(13.3)$ \\
\hline Body mass index $\left(\mathrm{kg} / \mathrm{m}^{2}\right)$, mean (SD) & $22.9(1.53)$ & $27.3(1.40)$ & $33.9(3.83)$ & $27.4(4.71)$ \\
\hline \multicolumn{5}{|l|}{ Smoking } \\
\hline Never & $85,608(59.7 \%)$ & $101,285(54.0 \%)$ & $54,809(51.5 \%)$ & $241,702(55.3 \%)$ \\
\hline Previous & $41,891(29.2 \%)$ & $67,116(35.8 \%)$ & $41,239(38.8 \%)$ & $150,246(34.4 \%)$ \\
\hline Current & $15,961(11.1 \%)$ & $19,162(10.2 \%)$ & $10,322(9.7 \%)$ & $45,445(10.4 \%)$ \\
\hline \multicolumn{5}{|l|}{ Alcohol intake } \\
\hline Daily or almost daily & $32,389(22.6 \%)$ & $40,452(21.6 \%)$ & $16,463(15.5 \%)$ & $89,304(20.4 \%)$ \\
\hline 3-4 times a week & $35,702(24.9 \%)$ & 46,235 (24.7\%) & $20,550(19.3 \%)$ & $10,2487(23.4 \%)$ \\
\hline Once or twice a week & $36,313(25.3 \%)$ & $49,273(26.3 \%)$ & $28,077(26.4 \%)$ & $113,663(26.0 \%)$ \\
\hline 1-3 times a month & $14,853(10.4 \%)$ & $19,717(10.5 \%)$ & $14,346(13.5 \%)$ & $48,916(11.2 \%)$ \\
\hline Special occasions only & $14,027(9.8 \%)$ & $18,826(10.0 \%)$ & $16,405(15.4 \%)$ & $49,258(11.3 \%)$ \\
\hline Never & $10,176(7.1 \%)$ & $13,060(7.0 \%)$ & $10,529(9.9 \%)$ & $33,765(7.7 \%)$ \\
\hline Fruit and vegetable intake (portion/day), mean (SD) & $2.01(0.825)$ & $1.95(0.827)$ & $1.94(0.832)$ & $1.97(0.828)$ \\
\hline Red meat (portion/week), mean (SD) & $1.93(1.38)$ & $2.14(1.42)$ & $2.28(1.53)$ & $2.11(1.44)$ \\
\hline Processed meat (portion/week), mean (SD) & $1.69(1.08)$ & $1.92(1.04)$ & $2.03(1.04)$ & $1.87(1.06)$ \\
\hline Oily fish (portion/week), mean (SD) & $1.65(0.919)$ & $1.65(0.921)$ & $1.59(0.946)$ & $1.64(0.927)$ \\
\hline Sedentary time (hours/day), mean (SD) & $4.48(2.03)$ & $5.12(2.22)$ & $5.64(2.51)$ & $5.03(2.28)$ \\
\hline Physical activity (hours/day), mean (SD) & $1.62(1.44)$ & $1.76(1.58)$ & $2.22(2.00)$ & $1.83(1.67)$ \\
\hline Diabetes at baseline & $2398(1.7 \%)$ & 7325 (3.9\%) & $11,485(10.8 \%)$ & $21,208(4.8 \%)$ \\
\hline Hypertension at baseline & $20,636(14.4 \%)$ & $48,570(25.9 \%)$ & $44,758(42.1 \%)$ & $11,3964(26.1 \%)$ \\
\hline
\end{tabular}

Data are presented as numbers (percentages) unless stated otherwise. Participants classified as underweight (BMl $<18.5 \mathrm{~kg} / \mathrm{m}^{2}$ were excluded from the analyses $(n=2629)$ 


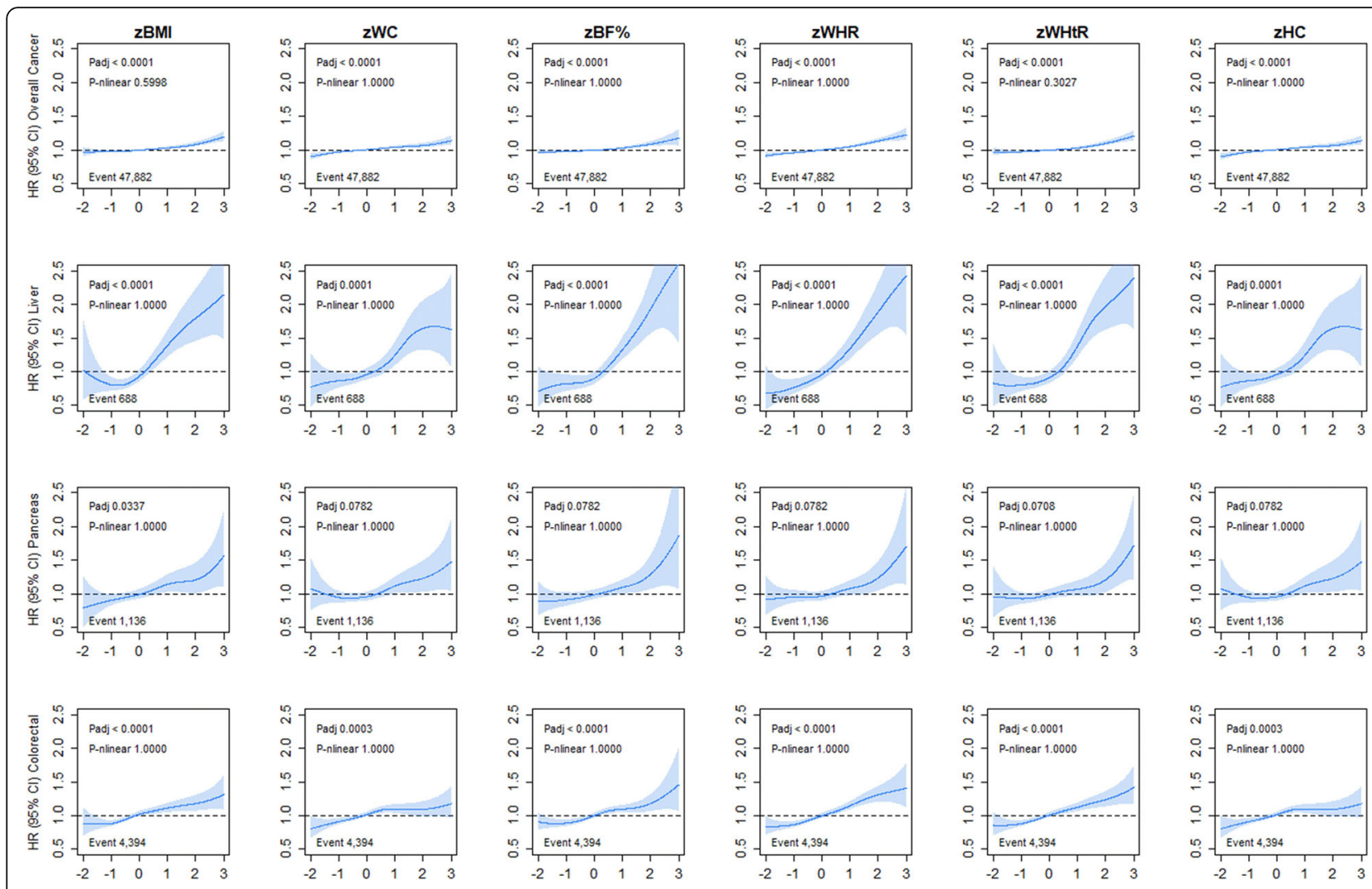

Fig. 1 Association of adiposity markers with overall, liver, pancreatic, and colorectal cancer incidence. Penalised splines were used to present the association between adiposity markers and cancer outcomes. The adiposity markers were sex-standardised to 1-SD increment. Analyses were adjusted for age, sex and ethnicity, education, deprivation, smoking, dietary intake (alcohol, fruits and vegetables, red and processed meat, and oily fish), physical activity and sedentary behaviour. BMI, body mass index; BF\%, body fat percentage; WHR, waist-hip ratio; WHtR, waist-height ratio; HC, hip circumference; HR, hazard ratio. Shaded areas represent $95 \%$ confidence intervals. All $P$ values were corrected for multiple testing by using the Holm's method. Participants classified as underweight (BMl $<18.5 \mathrm{~kg} / \mathrm{m}^{2}$ were excluded from the analyses $(n=2629)$

previously [27]. These were then used to calculate confidence intervals and $P$ values using normal approximation.

Competing risk due to non-cancer mortality was handled using a cause-specific model [28]. Participants who died due to non-cancer causes were marked as censored at their date of death. This approach was used instead of the sub distribution proportional hazards model because there is no evidence that the competing events influence the risk of cancer events, and because the current study aims to investigate associations rather than absolute risk.

Finally, because of potentially inflated type I errors due to multiple tests, all analyses were corrected for multiple testing using Holm's method [29], which performed similarly to Bonferroni's method while retaining higher statistical power [30]. The multiple testing corrected $P$ value are denoted as $P_{\text {adj }}$ for $P$ value for testing overall significance against no association, and $\mathrm{P}_{\text {nonlinear }}$ for $P$ value testing non-linearity.

All analyses were adjusted for age, sex, ethnicity, deprivation, education, smoking, alcohol consumption, intakes of fruit and vegetables, red and processed meat, oily fish, physical activity, and sedentary behaviours. Additionally, women-related cancer was further adjusted for hormonal replacement, age at menarche, and age at first and last live birth. Prostate cancer was additionally adjusted for family history of prostate cancer, and melanoma was further adjusted for sun exposure. All analyses were performed using R Statistical Software, version 3.6.2, with the package survival and pifpaf.

\section{Results}

This study included 437,393 participants who were followed up for 8.8 years (interquartile range (IQR) 7.9 to 9.6) for cancer incidence and 9.3 (IQR 8.6 to 9.9) for cancer mortality, after excluding the 2-year landmark analysis. Over this period, 47,882 incident cancer cases and 11,265 cancer deaths occurred (Additional file 1: Table S1 and S2). The characteristics of participants stratified by BMI categories are shown in Table 1. In 
summary, $53.8 \%$ of the study population were women, 94.6\% were of white European background. The mean population age was 56.3 years, $55.3 \%$ of subjects had never smoked, and $10.4 \%$ were current smokers.

Figure 1 shows the association of six adiposity markers with overall, liver, and colorectal cancer incidence. Although there was no evidence against linear associations with these cancer sites for all adiposity markers, the magnitude of association was higher for liver cancer incidence (HR ranging from 1.19 to 1.33 per 1-SD higher adiposity) compared with colorectal cancer (HR ranging from 1.07 to 1.13 per 1-SD higher adiposity), as shown in Additional file 1: Table S1. Similar results were found for overall, liver, pancreatic, and colorectal cancer mortality as shown in Additional file 1: Table S2. However, the association for WC and $\mathrm{HC}$ with colorectal cancer mortality was not significant (Additional file 1: Figure S2). Although a similar shape of association was observed for risk of pancreatic cancer incidence across all adiposity markers, only BMI was significantly associated with a higher risk after adjusting for multiple testing (Fig. 1). Similar results were observed for mortality from pancreatic cancer (Additional file 1: Figure S2). When the analyses were performed by segments of the digestive tract, distal, proximal, and colon cancer incidence were linearly associated with a higher risk across all adiposity markers (Additional file 1: Figure S3), but these associations were not observed for mortality (Additional file 1: Table S1 and Figure S4).

The association of adiposity markers with gallbladder and stomach (cardia and non-cardia) cancer incidence is shown in Fig. 2. There was no evidence of non-linear associations for gallbladder cancer across all six adiposity markers (HR varied from 1.28 to 1.50 per 1-SD higher adiposity). For stomach cancer incidence, a linear association was observed across all adiposity markers (HR ranged from 1.14 to 1.24 per 1-SD higher adiposity). However, when the analyses were stratified by stomach cardia and non-cardia, only stomach cardia was linearly associated with all adiposity markers (HR varied from 1.25 to 1.35 per 1 -SD higher adiposity)

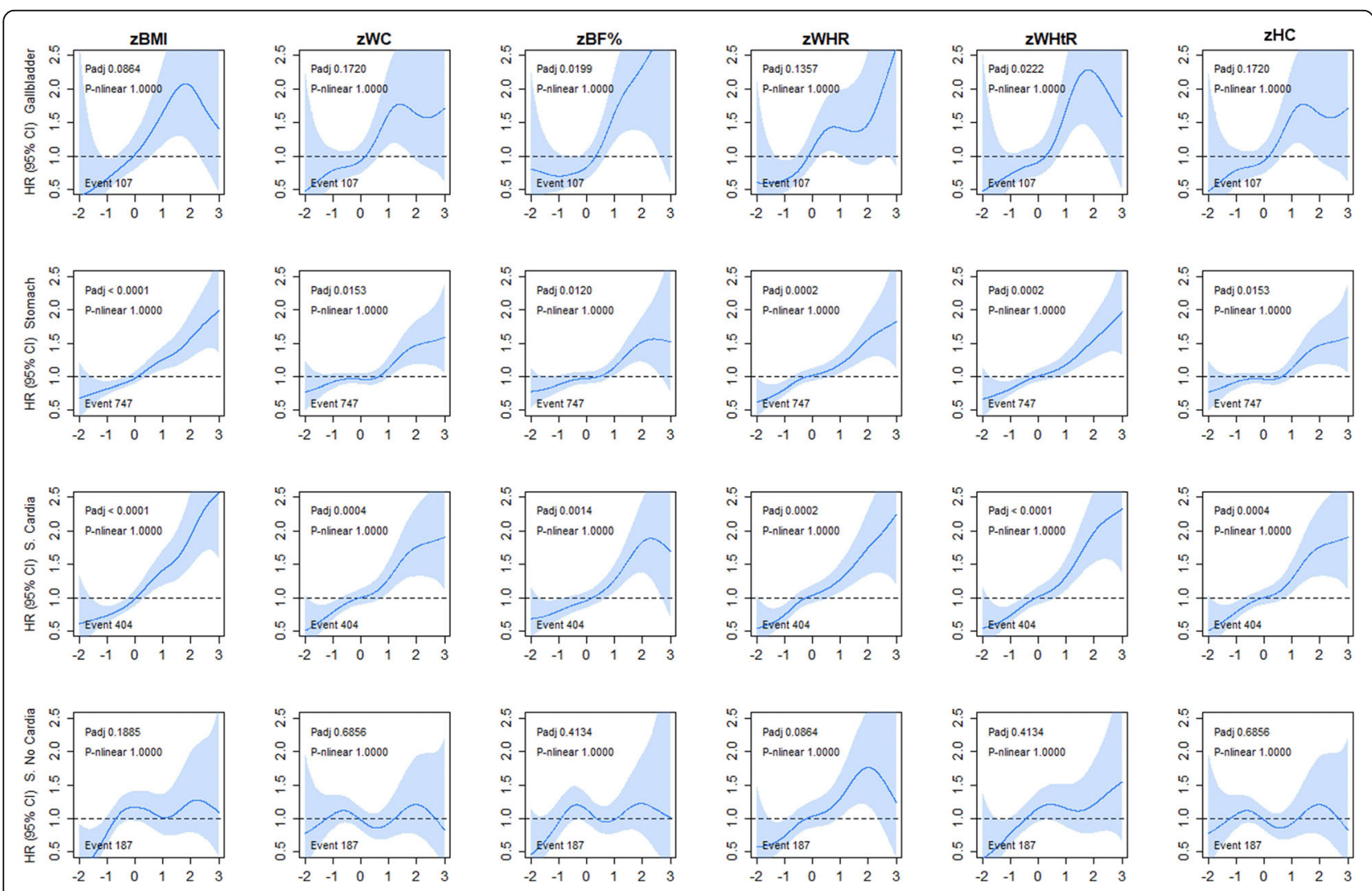

Fig. 2 Association of adiposity markers with gallbladder and stomach cancer incidence. Penalised splines were used to present the association between adiposity markers and cancer outcomes. The adiposity markers were sex-standardised to 1-SD increment. Analyses were adjusted for age, sex and ethnicity, education, deprivation, smoking, dietary intake (alcohol, fruits and vegetables, red and processed meat, and oily fish), physical activity and sedentary behaviour. BMI, body mass index; BF\%, body fat percentage; WHR, waist-hip ratio; WHtR, waist-height ratio; HC, hip circumference; HR, hazard ratio. Shaded areas represent $95 \%$ confidence intervals. All $P$ values were corrected for multiple testing by using the Holm's method. Participants classified as underweight $\left(\mathrm{BMl}<18.5 \mathrm{~kg} / \mathrm{m}^{2}\right.$ were excluded from the analyses $(\mathrm{n}=2629)$ 


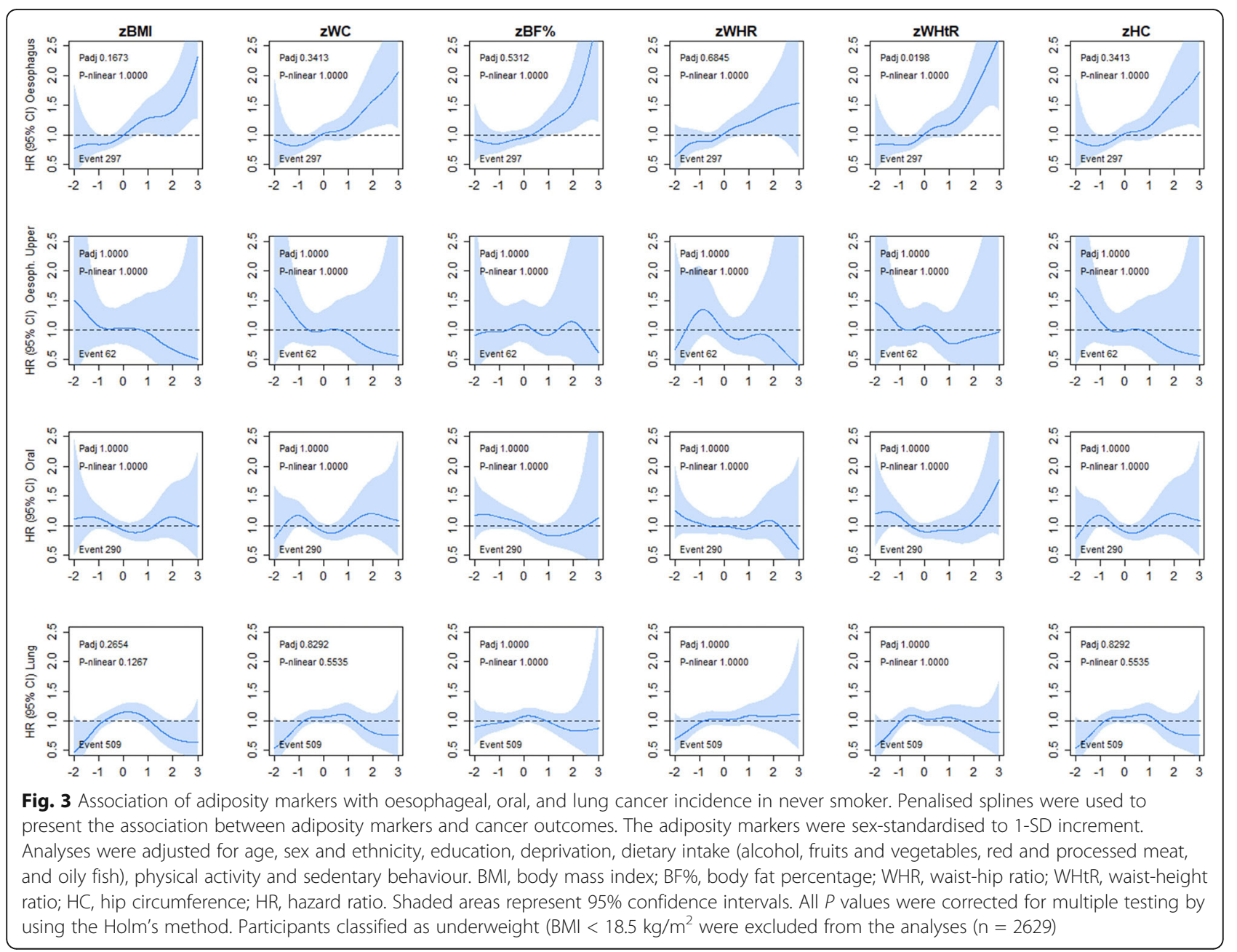

(Additional file 1: Table S1). Similar patterns of associations were observed for mortality from gallbladder, stomach, and stomach cardia cancer (Additional file 1: Figure S5).

The associations between adiposity and respiratoryrelated cancers in never smokers are shown in Fig. 3. Although similar shaped associations were observed for oesophageal cancer incidence across all adiposity markers, only WHtR was significant (HR ranged from 1.19 to 1.26 per 1 -SD higher adiposity) (Additional file 1: Table S1). Similar associations were observed for oesophageal cancer mortality (Additional file 1: Figure S6). No associations were observed for upper oesophageal, oral, and lung cancer incidence and mortality across any of the adiposity markers.

Lymphatic cancer was linearly associated with BMI, WC, and $\mathrm{HC}$, for incidence (HR ranged from 1.06 to 1.08 per $1-\mathrm{SD}$ higher adiposity, Additional file 1: Table S1). However, no association were observed for leukaemia, non-Hodgkin and myeloma cancer incidence and mortality across any of the adiposity markers (Fig. 4 and Additional file 1: Figure S7).

For sex-specific cancers, we observed a steeper linear association for uterine (HR ranged from 1.26 to 1.70) and endometrial (HR ranged from 1.29 to 1.78 ) cancer incidence (Fig. 5 and Additional file 1: Table $\mathrm{S} 1)$. The strongest magnitude of association for both uterine and endometrial cancer incidence was observed for BF\% whereas WHR shows the smallest magnitude of association of any adiposity marker (Additional file 1: Table S1). Although similar associations were observed for uterine and endometrial cancer mortality across all adiposity markers, mortality from cervical cancer showed a borderline U-shaped association with BMI, WC, BF\%, WHtR, and HC (Additional file 1: Figure S9 and Table S2). No association was found between adiposity and ovarian cancer incidence and mortality. For breast cancer incidence, a linear association was observed for BMI, BF\%, WHtR, and WHR; however, a slight departure from 


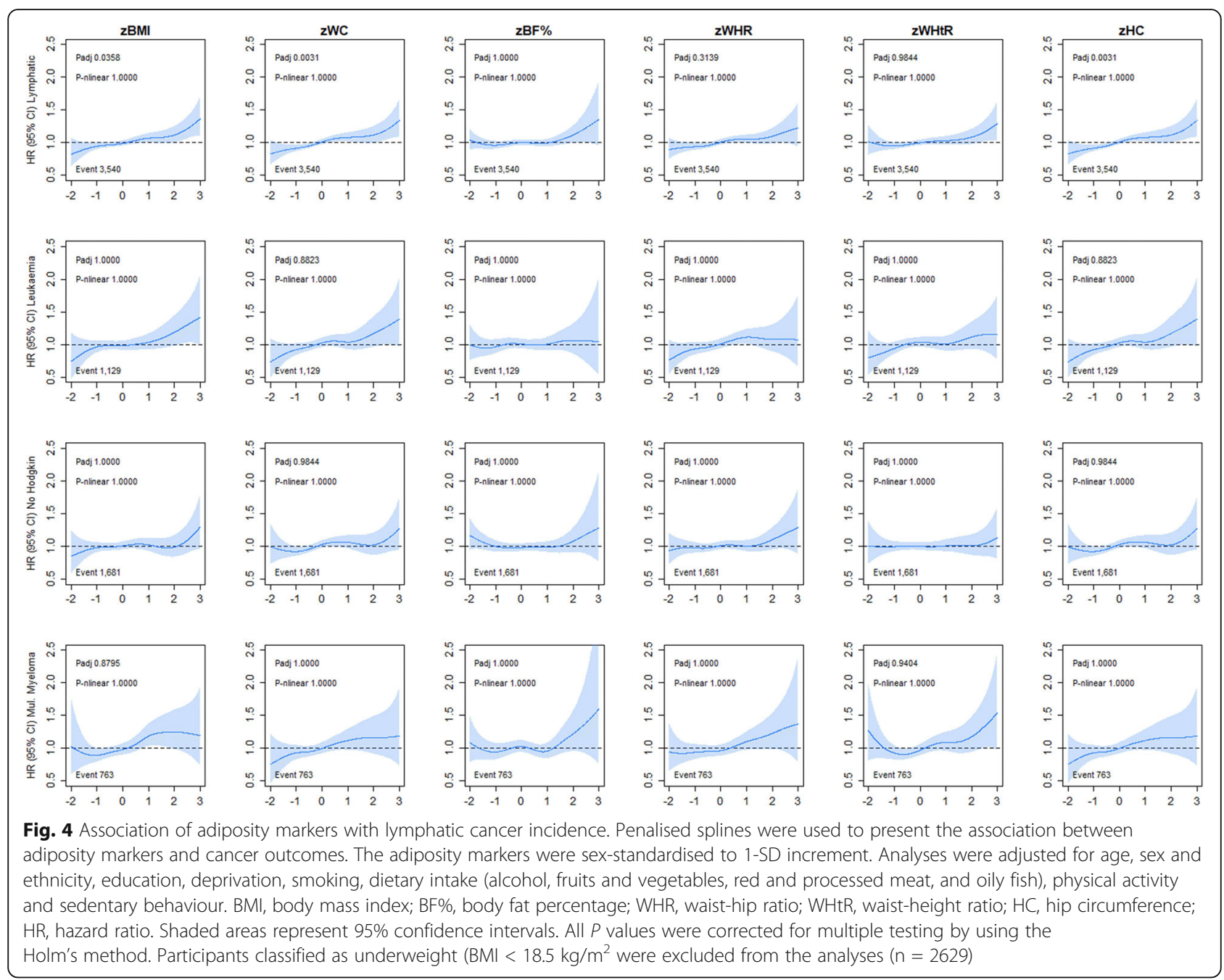

linearity was observed for WC and HC (Fig. 6). When the analyses were stratified into pre and post menopause, the adiposity markers were associated with breast cancer incidence in postmenopausal women only (Fig. 6). No associations were observed for breast cancer mortality (Additional file 1: Figure S10). The associations for women-related cancers remained largely unchanged when the analyses were further adjusted for use of hormonal replacement therapy, age at menarche, and age at first and date of last live birth (Additional file 1: Figure S8, S9 and S11). For men, only prostate cancer incidence, but not mortality, was inversely associated with WC and HC (Fig. 6 and Additional file 1: Figure S10).

Kidney cancer incidence and mortality were linearly associated with all adiposity markers, with HR ranging from 1.18 to 1.27 per 1-SD higher adiposity (Fig. 7 and Additional file 1: Figure S12 and Table S1). For bladder cancer, we observed a higher risk of cancer incidence only at the higher end of the BMI and WHtR ranges (Fig. 7). However, these associations were not observed for bladder cancer mortality (Additional file 1: Figure S12). For melanoma cancer incidence, only WC and $\mathrm{HC}$ were linearly associated with a higher risk (Fig. 7).

Our PAF analyses show that the proportions of cancer attributable to BMI vary considerably by cancer site. Endometrial, uterine, and gallbladder were the top three cancers for which obesity accounted for $43.8 \%, 39.2 \%$, and $29.9 \%$ incident cases and $63.8 \%, 46.1 \%$, and $39.8 \%$ of deaths, respectively (Fig. 8). When the predictive ability of BMI was compared with the other adiposity markers using $\mathrm{C}$-index, there were no evidence of a significant improvement in $\mathrm{C}$-indices from models using $\mathrm{WC}, \mathrm{BF} \%$, WHR, WHtR, and HC over the model with BMI (Additional file 1: Table S3). The associations for overall, liver, kidney, stomach, pancreatic, bladder, gallbladder, colorectal cancer, endometrium, uterine, and breast (postmenopausal in women) cancer remained significant 


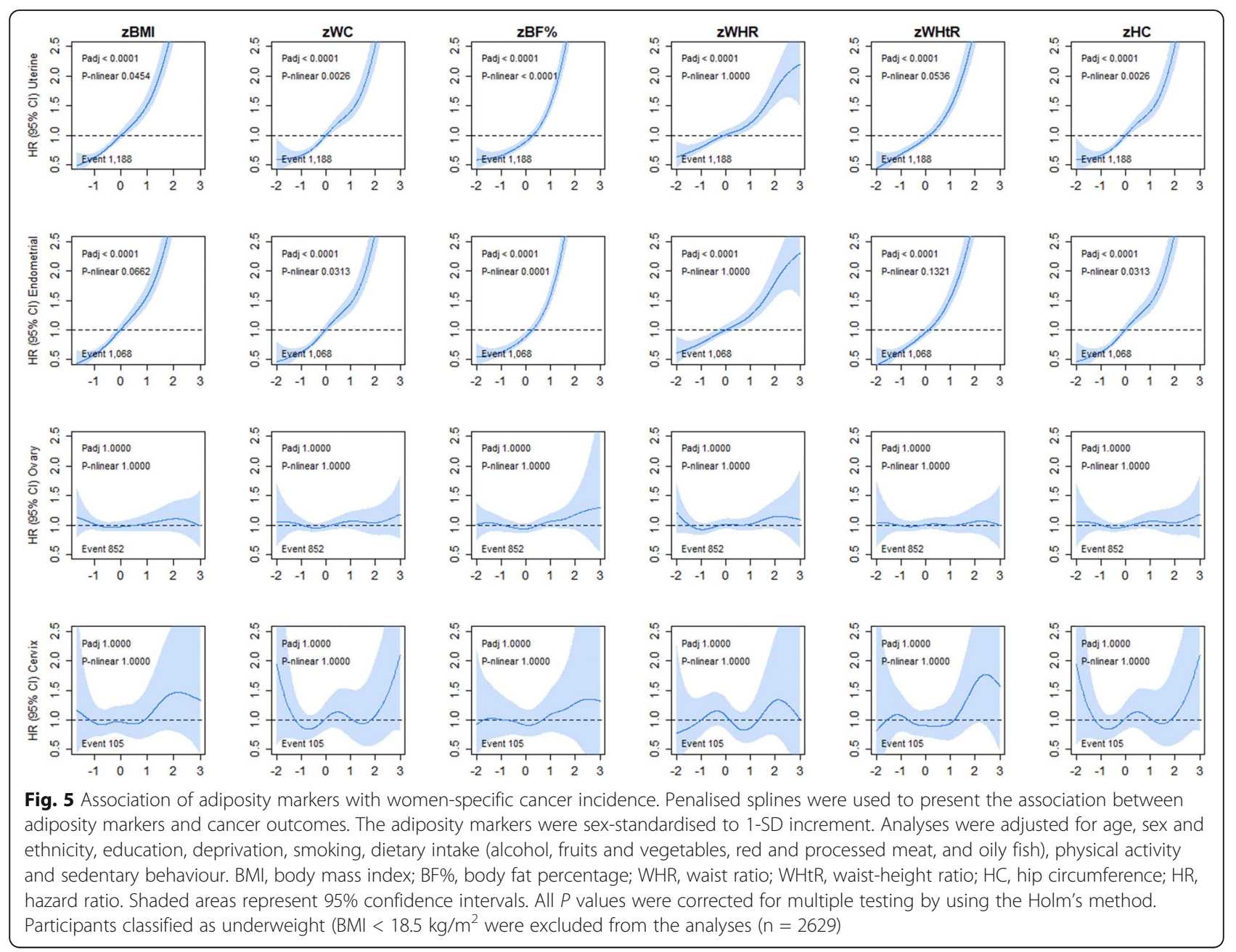

and largely unchanged when the analyses were adjusted for competing events (Additional file 1: Table S4).

When we conducted the analyses including underweight people, the association between adiposity and cancer remained linear (Additional file 1: Figure S13 and Figure S14). Similar results were found for cancer incidence and mortality when we added height as a covariate; some associations were slightly stronger as was the association between BMI and overall cancer incidence and mortality; (Additional file 1: Table S5 and Table S6).

\section{Discussion}

This study provides important evidence regarding the risk of 24 cancer sites associated with multiple adiposity markers. Higher levels of adiposity, regardless of the adiposity marker used, were associated in a linear manner with a higher incidence of liver, kidney, stomach, pancreatic, bladder, gallbladder, colorectal cancer, endometrial, uterine, and breast (in postmenopausal women) cancer. If the associations observed were causal, reducing the BMI of obese individuals to the normal range could prevent $43.8 \%, 39.2 \%$, and $29.9 \%$ of incidence and $63.8 \%$, $46.1 \%$, and $39.8 \%$ deaths from endometrial, uterine, and gallbladder cancers, respectively.

Our findings corroborate previous evidence, including the WCRF obesity and cancer 2018 report and meta-analyses from protective cohort studies [31-34], that adult adiposity (assessed using BMI) is associated with higher risk of oesophageal, pancreatic, liver, colorectal, postmenopausal breast, and endometrial cancers. Furthermore, our findings add strength to previously weak evidence of links between BMI and stomach cancer risk [35]. On the other hand, our findings did not find evidence for an association between BMI (and any other markers of adiposity) and ovarian cancer as reported by others [36], which could be attributed to our comprehensive confounder adjustments. We also found inverse 


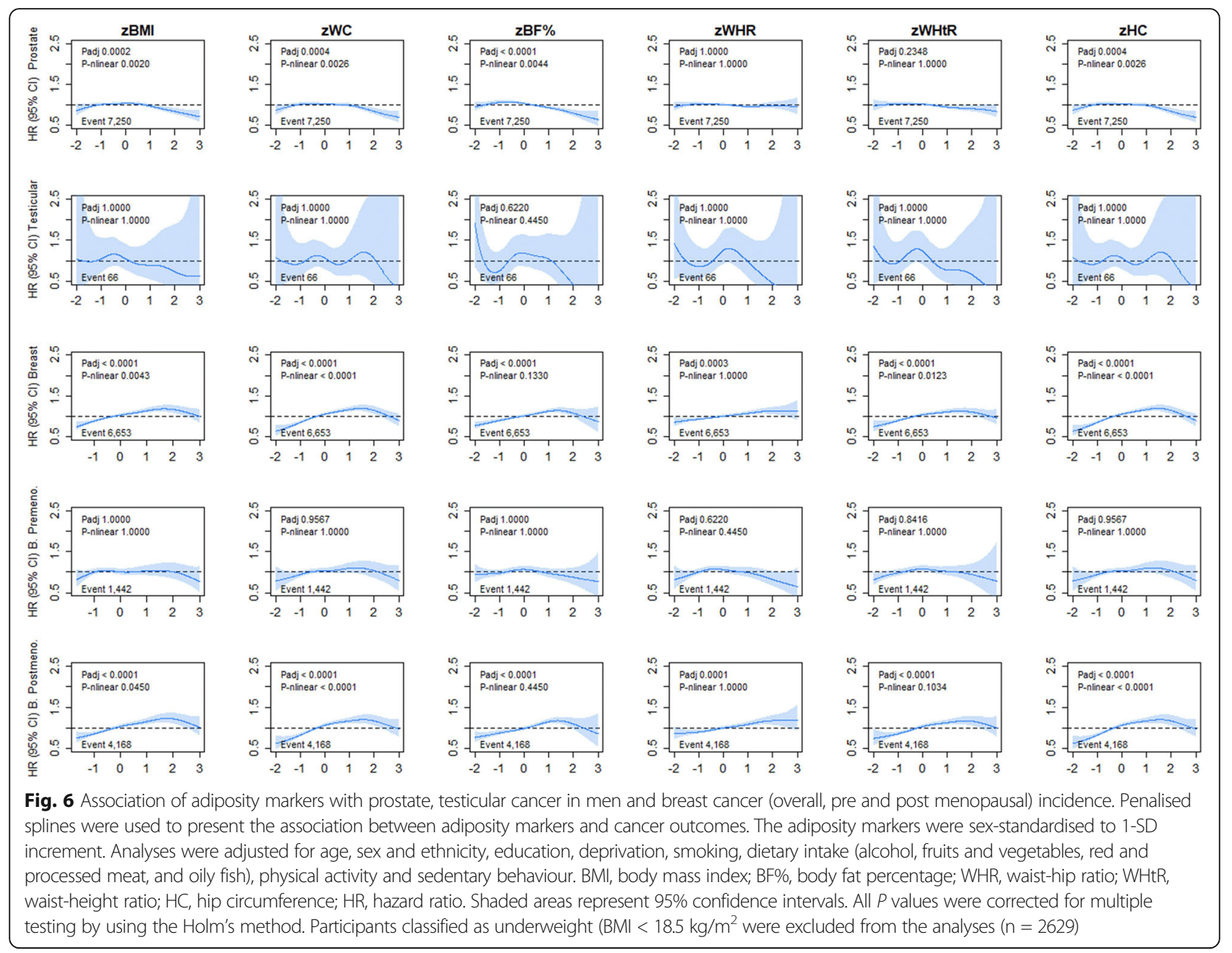

associations between five adiposity markers and risk of prostate cancer. Although excess adiposity has been associated with multiple cancers, evidence of its association with prostate cancer has been restricted to advanced prostate cancer only [37, 38]. However, a recent systematic review of data from 78 studies, including a meta-analysis of 67 studies, reported no association between BMI and prostate cancer [37, 38]. These authors also concluded that previously reported inverse associations between BMI and prostate cancer may be due to incomplete diagnosis (not all men being biopsied). The assumption that men who have not been tested for prostate do not have prostate cancer may lead to bias and inverse associations [37]. BMI and WHtR were positively associated with bladder cancer, in concordance with the meta-analysis of 15 cohort studies, published by Sun et al., which showed a linear association between adiposity and bladder cancer [39].
We did not find a significant association between adiposity and lung cancer in never smokers. These disagree with a recent meta-analysis with considerable statistical power, which pooled data from 29 observational studies, including 15 million never smokers, where BMI was inversely associated with lung cancer [40].

There is convincing evidence [41] that greater adiposity is associated with increased risk of colorectal cancer, assessed mainly as BMI in prospective cohort studies [7, 35, 41-44]. Our study corroborates these findings and adds novel evidence that other adiposity markers are also consistently associated with an increased risk of colorectal cancer. We also observed that all adiposity markers were positively associated with higher liver cancer risk with broadly consistent effect sizes. Furthermore, we found that all adiposity markers were associated with an increased risk of breast cancer. But the association appeared to occur in postmenopausal 


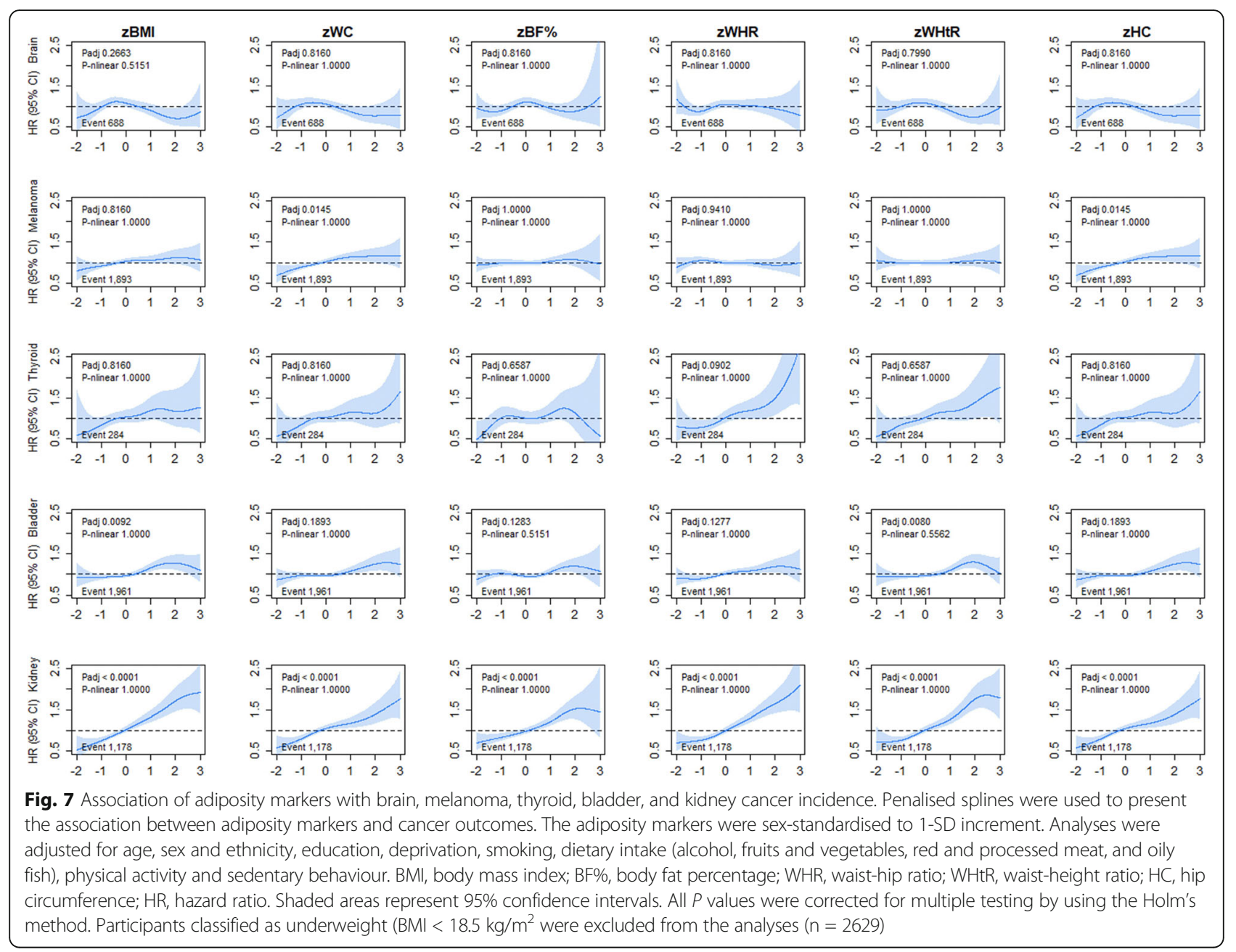

women only. These findings confirm previous evidence from prospective cohort studies $[33,45,46]$.

\section{Implications of findings}

The findings of this study have important clinical implications. First, it provides evidence that central (waist and hip circumference) and overall adiposity (BMI and BF\%) markers produced similar relative risk estimates. Therefore, the use of BMI, a simple and low-cost measurement, is adequate for clinical screening in terms of cancer risk, and there is no advantage in using more complicated or more expensive measures such as $\mathrm{WC}$ or $\mathrm{BF} \%$. We also found that a significant proportion of cancers could be prevented by reducing obesity, especially liver and kidney cancer in men and endometrial and uterine cancer in women.

\section{Strengths and Limitations}

UK Biobank is not a representative sample of the UK older adult population, so we should be cautious in generalising summary statistics to the general population. However, relative risks derived from UK Biobank are consistent with more representative population cohorts [47]. The adiposity exposures used in the study were measured by trained staff using standardised protocols; therefore, this minimises the chance of measurement error and misclassification. However, there are several limitations that should be taken into account. Reverse causation is a concern in prospective cohort studies investigating the association between adiposity and cancer. However, to minimise the effect of reverse causation in our study, we excluded all participants with cancers diagnosed within the first 2 years of follow-up. Residual confounding is also possible even though we have adopted a comprehensive adjustment scheme. In addition, although we used data from hospital admission and deaths registers, available in the UK, we cannot exclude misclassification for cancer-specific sites or uncommon cancers. Although UK Biobank is a large observational study, some cancers had limited numbers of events, which limited our power to identify some associations with adiposity markers. 


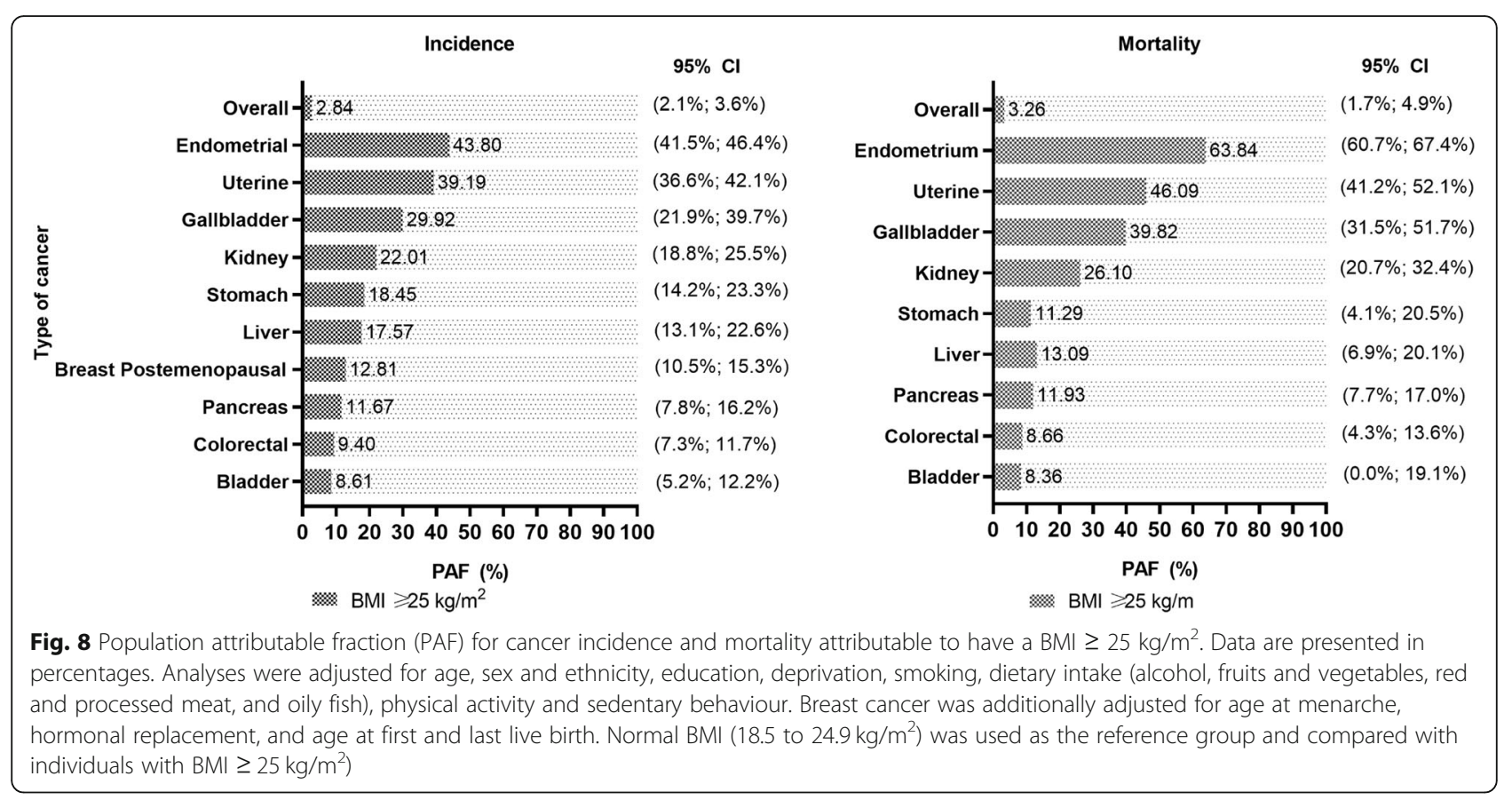

\section{Conclusion}

Adiposity, regardless of the marker used, was associated with an increased risk of 10 cancer sites. Furthermore, the associations were mostly linear among all adiposity markers. We found no evidence that the use of other adiposity markers, such as central adiposity or body fat, improves the prediction ability for cancer risk beyond the risk attributable to BMI.

\section{Supplementary information}

The online version contains supplementary material available at https://doi. org/10.1186/s12916-020-01848-8.

Additional file 1: Figure S1. Formula for PAFs. Figure S2. Association of Adiposity markers with overall, liver, pancreas and colorectal cancer mortality. Figure S3. Association of Adiposity markers with colorectal cancer incidence. Figure S4. Association of Adiposity markers with colorectal cancer mortality. Figure S5. Association of Adiposity markers with gallbladder and stomach cancer mortality. Figure S6. Association of Adiposity markers oesophagus, oral and lung cancer mortality in no smokers. Figure S7. Association of Adiposity markers with lymphatic cancer mortality. Figure S8. Association of Adiposity markers with uterine, endometrial, ovary and cervical cancer incidence adjusted. Figure S9. Association of Adiposity markers with uterine, endometrial, ovary and cervical cancer mortality. Figure S10. Association of Adiposity markers with prostate, testicular cancer in men and breast cancer in postmenopausal women mortality. Figure S11. Association of adiposity markers with prostate, testicular, and breast cancer incidence additionally adjusted for sex-related covariates. Figure S12. Association of adiposity markers with brain, melanoma, thyroid, bladder and kidney cancer mortality. Figure S13. Association of Adiposity markers with overall, liver, pancreas, colorectal cancer and stomach cardia incidence with underweight people. Figure S14. Association of Adiposity markers with gallbladder, bladder, kidney, breast and endometrium cancer incidence with underweight people. Table S1. Association of adiposity markers with incidence from 24 cancer sites per 1 SD increase in adiposity markers.

Table S2. Association of adiposity markers with mortality from 24 cancer sites per 1 SD increase in adiposity markers. Table S3. C-Index for the predictive ability of BMI versus other adiposity markers. Table S4. Association of adiposity markers with incidence from 24 cancer sites after accounting for competing risk. Table S5. Association of adiposity markers with incidence from 24 cancer sites per 1 SD increase in adiposity markers with height as covariate. Table S6. Association of adiposity markers with mortality from 24 cancer sites per 1 SD increase in adiposity markers with height as covariate.

\section{Abbreviations}

95\% Cl: 95\% confidence intervals; BF\%: Body fat percentage; BFl: Body fat mass index; BMI: Body mass index; HC: Hip circumference; HR: Hazard ratio; Padj: $P$ value for linear association adjusted for multiple testing; $P$-nlinear: $P$ value for non-linear association adjusted for multiple testing; SD: Standard deviation; WC: Waist circumference; WHR: Waist-hip ratio; WHtR: Waist-height ratio

\section{Acknowledgements}

We thank UK Biobank participants. This research has been conducted using the UK Biobank Resource (application 7155).

\section{Authors' contributions}

The authors' responsibilities were as follows-S.P-S, J.C.M, F. H, J.P.P, and C.C$\mathrm{M}$ and contributed to the conception and design of the study, advised on all statistical aspects, and interpreted the data. S.P-S, F. H, and C.C-M performed the statistical analysis. S.P-S, E.S.C, L.F.M.R, K. F, F.H, J.C.M, JPP, and C.C-M interpreted findings and drafted the manuscript. All authors read and approved the final manuscript. S.P-S, C.C-M, and J.P.P had full access to all the data in the study and take responsibility for the integrity of the data and the accuracy of the data analysis.

\section{Funding}

The UK Biobank was supported by the Wellcome Trust, Medical Research Council, Department of Health, Scottish Government and the Northwest Regional Development Agency. It has also had funding from the Welsh Assembly Government and the British Heart Foundation. S.P.-S. receive financial support from the Chilean Government for doing their PhD (ANIDBecas Chile). The research was designed, conducted, analysed, and interpreted by the authors entirely independently of the funding sources. The sponsor had no role in any aspect of the study's design, data collection, analysis, and writing up. The corresponding authors had full access to all the 
data in the study and had final responsibility for the decision to submit for publication.

\section{Availability of data and materials}

The data that support the findings of this study are available from UK Biobank but restrictions apply to their availability. These data were used under licence for the current study and so are not publicly available. The data are, however, available from the authors upon reasonable request and with permission of UK Biobank.

\section{Ethics approval and consent to participate}

This study was performed under generic ethical approval obtained by UK Biobank from the National Health Service National Research Ethics Service (approval letter ref. 11/NW/0382, 17 June 2011). All participants provided written informed consent to participate in the UK Biobank study. The study protocol is available online (http://www.ukbiobank.ac.uk/wp-content/ uploads/2018/05/Favourable-Ethical-Opinion-and-RTB-Approval-16.NW_.02 74-200778-May-2016.pdf).

\section{Competing interests}

No to declare.

\section{Author details}

Institute of Health and Wellbeing, University of Glasgow, Glasgow G12 8RZ, UK. ${ }^{2}$ Institute of Cardiovascular and Medical Sciences, University of Glasgow, Glasgow G12 8TA, UK. ${ }^{3}$ Departamento de Medicina Preventiva, Universidade Federal de São Paulo, Escola Paulista de Medicina, Sao Paulo, Brazil. ${ }^{4}$ Advanced Center for Chronic Diseases, Universidad de Chile and Pontificia Universidad Católica de Chile, Santiago, Chile. ${ }^{5}$ Facultad de Medicina, Pontificia Universidad Católica de Chile, Santiago, Chile. ${ }^{6}$ Human Nutrition Research Centre, Population Health Sciences Institute, Newcastle University, Newcastle upon Tyne NE2 4HH, UK. ${ }^{7}$ Centro de Investigación en Fisiología del Ejercicio (CIFE), Universidad Mayor, 7510041 Santiago, Chile. ${ }^{8}$ Laboratorio de Rendimiento Humano, Grupo de Estudio en Educación, Actividad Física y Salud (GEEAFyS), Universidad Católica del Maule, 3480112 Talca, Chile.

\section{Received: 29 June 2020 Accepted: 9 November 2020}

\section{Published online: 11 January 2021}

\section{References}

1. Mokdad AH, Ford ES, Bowman BA, Dietz WH, Vinicor F, Bales VS, et al. Prevalence of obesity, diabetes, and obesity-related health risk factors, 2001. Jama-J Am Med Assoc. 2003;289(1):76-9.

2. Bhaskaran K, Douglas I, Forbes H, dos-Santos-Silva I, Leon DA, Smeeth L. Body-mass index and risk of 22 specific cancers: a population-based cohort study of 5.24 million UK adults. Lancet. 2014;384(9945):755-765.

3. World Cancer Research Fund. Continuous Update Project Expert Report 2018. Body fatness and weight gain and the risk of cancer 2018.

4. Freisling H, Arnold M, Soerjomataram I, O'Doherty MG, Ordonez-Mena JM, Bamia C, et al. Comparison of general obesity and measures of body fat distribution in older adults in relation to cancer risk: meta-analysis of individual participant data of seven prospective cohorts in Europe. $\mathrm{Br}$ J Cancer. 2017:116(11):1486-97.

5. Keimling M, Renehan AG, Behrens G, Fischer B, Hollenbeck AR, Cross AJ, et al. Comparison of associations of body mass index, abdominal adiposity, and risk of colorectal cancer in a large prospective cohort study. Cancer Epidemiol Biomark Prev. 2013;22(8):1383-94.

6. Wang L, Jin G, Yu C, Lv J, Guo Y, Bian Z, et al. Cancer incidence in relation to body fatness among 0.5 million men and women: findings from the China Kadoorie Biobank. Int J Cancer. 2020;146(4):987-98.

7. Lee KR, Seo MH, Do Han K, Jung J, Hwang IC. Waist circumference and risk of 23 site-specific cancers: a population-based cohort study of Korean adults. Br J Cancer. 2018;119(8):1018-27.

8. Guo W, Key TJ, Reeves GK. Accelerometer compared with questionnaire measures of physical activity in relation to body size and composition: a large cross-sectional analysis of UK Biobank. BMJ Open. 2019:9(1):e024206.

9. Ortega LS, Bradbury KE, Cross AJ, Morris JS, Gunter MJ, Murphy N. A prospective investigation of body size, body fat composition and colorectal cancer risk in the UK Biobank. Sci Rep. 2017;7(1):17807.
10. Perez-Cornago A, Key TJ, Allen NE, Fensom GK, Bradbury KE, Martin RM, et al. Prospective investigation of risk factors for prostate cancer in the UK Biobank cohort study. Br J Cancer. 2017;117(10):1562-71.

11. Omiyale W, Allen NE, Sweetland S. Body size, body composition and endometrial cancer risk among postmenopausal women in UK Biobank. Int J Cancer. 2020;147:2405-15.

12. Barberio AM, Alareeki A, Viner B, Pader J, Vena JE, Arora P, et al. Central body fatness is a stronger predictor of cancer risk than overall body size. Nat Commun. 2019;10:383.

13. Ross R, Neeland IJ, Yamashita S, Shai I, Seidell J, Magni P, et al. Waist circumference as a vital sign in clinical practice: a consensus statement from the IAS and ICCR Working Group on Visceral Obesity. Nat Rev Endocrinol. 2020;16(3):177-89.

14. World Cancer Research Fund. Continuous Update Project Expert Report 2018. Meat, fish and dairy products and the risk of cancer 2018.

15. Aune D, Greenwood DC, Chan DS, Vieira R, Vieira AR, Navarro Rosenblatt DA, et al. Body mass index, abdominal fatness and pancreatic cancer risk: a systematic review and non-linear dose-response meta-analysis of prospective studies. Ann Oncol. 2012;23(4):843-52.

16. Collins R. What makes UK Biobank special? Lancet. 2012;379(9822):1173-4.

17. Sudlow C, Gallacher J, Allen N, Beral V, Burton P, Danesh J, et al. UK Biobank: an open access resource for identifying the causes of a wide range of complex diseases of middle and old age. PLoS Med. 2015;12(3):e1001779.

18. Palmer LJ. UK Biobank: bank on it. Lancet. 2007;369(9578):1980-2.

19. World Health Organization. Obesity: preventing and managing the global epidemic. Report of a WHO consultation. World Health Organization technical report series, Geneva. 2000.

20. Townsend P, Phillimore M, A B. Health and deprivation: inequality and the North. London. Ltd CH, editor1988.

21. IPAQ. Version 2 April Internet. Available from: www.ipaq.ki.se. Accessed 20 Oct 2020.

22. Govindarajulu US, Malloy EJ, Ganguli B, Spiegelman D, Eisen EA. The comparison of alternative smoothing methods for fitting non-linear exposure-response relationships with Cox models in a simulation study. Int J Biostat. 2009;5(1):Article 2

23. Cabinet Secretary for Health and Sport. The Scottish Health Survey 2018 main report - revised 2020: 21 Feb 2020; 2020 [Available from: https://www. gov.scot/publications/scottish-health-survey-2018-volume-1-main-report/.

24. Lifestyles Team ND. Health survey for England: NHS Digital, part of the government statistical service; 2019 [updated 3 Dec 2019. Available from: https://files.digital.nhs.uk/52/FD7E18/HSE18-Adult-Child-Obesity-rep.pdf.

25. National Survey for Wales. National Survey for Wales 2018-19: Adult lifestyle 2020 [Available from: https://gov.wales/sites/default/files/statistics-andresearch/2019-06/national-survey-for-wales-april-2018-to-march-2019-adultlifestyle-534.pdf

26. Vander Hoorn S, Ezzati M RA, Anthony Rodgers, Alan D. Lopez, Murray CJL. Estimating attributable burden of disease from exposure and hazard data. In: Organization GWH, editor. Comparative quantification of health risks: global and regional burden of disease attributable to selected major risk factor 2004. 2129-40.

27. Hanley JA, Mcneil BJ. The meaning and use of the area under a receiver operating characteristic (roc) curve. Radiology. 1982;143(1):29-36.

28. Lau B, Cole SR, Gange SJ. Competing risk regression models for epidemiologic data. Am J Epidemiol. 2009;170(2):244-56.

29. ETH Zurich. Adjust P-values for multiple comparisons [cited 2020 05-182020]. Available from: https://stat.ethz.ch/R-manual/R-devel/library/stats/ html/p.adjust.html.

30. Holm S. A simple sequentially rejective multiple test procedure. Scand J Stat. 1979:6:65-70.

31. Fang XX, Wei JY, He XY, Lian J, Han D, An P, et al. Quantitative association between body mass index and the risk of cancer: a global meta-analysis of prospective cohort studies. Int J Cancer. 2018;143(7):1595-603.

32. Wei L, Li N, Wang G, Feng X, Lyu Z, Li X, et al. Waist circumference might be a predictor of primary liver cancer: a population-based cohort study. Front Oncol. 2018;8:607.

33. Renehan AG, Tyson M, Egger M, Heller RF, Zwahlen M. Body-mass index and incidence of cancer: a systematic review and meta-analysis of prospective observational studies. Lancet. 2008:371(9612):569-78.

34. Stolzenberg-Solomon RZ, Schairer C, Moore S, Hollenbeck A, Silverman DT. Lifetime adiposity and risk of pancreatic cancer in the NIH-AARP Diet and Health Study cohort. Am J Clin Nutr. 2013;98(4):1057-65. 
35. Kubo A, Corley DA. Body mass index and adenocarcinomas of the esophagus or gastric cardia: a systematic review and meta-analysis. Cancer Epidemiol Biomark Prev. 2006;15(5):872-8.

36. Kyrgiou M, Kalliala I, Markozannes G, Gunter MJ, Paraskevaidis E, Gabra H, et al. Adiposity and cancer at major anatomical sites: umbrella review of the literature. BMJ. 2017;356:j477.

37. Harrison S, Tilling K, Turner EL, Martin RM, Lennon R, Lane JA, et al. Systematic review and meta-analysis of the associations between body mass index, prostate cancer, advanced prostate cancer, and prostatespecific antigen. Cancer Causes Control. 2020;31(5):431-49.

38. Lauby-Secretan B, Scoccianti C, Loomis D, Grosse Y, Bianchini F, Straif K, et al. Body fatness and Cancer - viewpoint of the IARC Working Group. N Engl J Med. 2016:375(8):794-8.

39. Sun JW, Zhao LG, Yang Y, Ma X, Wang YY, Xiang YB. Obesity and risk of bladder cancer: a dose-response meta-analysis of 15 cohort studies. PLoS One. 2015;10(3):e0119313.

40. Zhu H, Zhang S. Body mass index and lung cancer risk in never smokers: a meta-analysis. BMC Cancer. 2018;18(1):635.

41. Johnson CM, Wei C, Ensor JE, Smolenski DJ, Amos Cl, Levin B, et al. Metaanalyses of colorectal cancer risk factors. Cancer Causes Control. 2013;24(6): $1207-22$.

42. Jarvis D, Mitchell JS, Law PJ, Palin K, Tuupanen S, Gylfe A, et al. Mendelian randomisation analysis strongly implicates adiposity with risk of developing colorectal cancer. Br J Cancer. 2016;115(2):266-72.

43. Guo Y, Warren Andersen S, Shu XO, Michailidou K, Bolla MK, Wang Q, et al Genetically predicted body mass index and breast cancer risk: Mendelian randomization analyses of data from 145,000 women of European descent. PLoS Med. 2016;13(8):e1002105.

44. Thrift AP, Gong J, Peters U, Chang-Claude J, Rudolph A, Slattery ML, et al. Mendelian randomization study of body mass index and colorectal cancer risk. Cancer Epidemiol Biomark Prev. 2015;24(7):1024-31.

45. Benn M, Tybjaerg-Hansen A, Smith GD, Nordestgaard BG. High body mass index and cancer risk-a Mendelian randomisation study. Eur J Epidemiol. 2016;31(9):879-92.

46. Guo W, Key TJ, Reeves GK. Adiposity and breast cancer risk in postmenopausal women: results from the UK Biobank prospective cohort. Int J Cancer. 2018;143(5):1037-46.

47. Batty GD, Gale CR, Kivimäki M, Deary IJ, Bell S. Comparison of risk factor associations in UK Biobank against representative, general population based studies with conventional response rates: prospective cohort study and individual participant meta-analysis. BMJ. 2020;368:m131

\section{Publisher's Note}

Springer Nature remains neutral with regard to jurisdictional claims in published maps and institutional affiliations.

Ready to submit your research? Choose BMC and benefit from:

- fast, convenient online submission

- thorough peer review by experienced researchers in your field

- rapid publication on acceptance

- support for research data, including large and complex data types

- gold Open Access which fosters wider collaboration and increased citations

- maximum visibility for your research: over $100 \mathrm{M}$ website views per year

At $\mathrm{BMC}$, research is always in progress.

Learn more biomedcentral.com/submissions 